\title{
Bremst die Doppik öffentliche Investitionen? Ergebnisse aus drei aktuellen Evaluationsstudien
}

\author{
Internationale Organisationen empfehlen nachdrücklich, öffentliche Haushalte von der \\ klassischen kameralen Buchführung auf die doppelte Buchführung (Doppik) umzustellen. \\ Doppische Haushalte sollen den Wertverzehr der öffentlichen Infrastruktur offenlegen und die \\ implizite Verschuldung sichtbar machen. Drei unabhängig voneinander entstandene Studien \\ haben die Wirkung der Doppik am Beispiel deutscher Kommunen evaluiert und kommen \\ zu teils überraschenden Ergebnissen. Doppisch buchende Kommunen veräußern zwar wie \\ erwartet weniger öffentliches Vermögen, sie investieren aber tendenziell auch weniger in \\ neues Vermögen. Diese empirischen Ergebnisse nähren Zweifel, ob die doppelte Buchführung \\ tatsächlich nachhaltigere Haushaltsentscheidungen fördert.
}

Die Ablösung der Kameralistik durch die doppelte Buchführung (Doppik) ist die wohl umfassendste Reform der öffentlichen Haushaltsführung der letzten Jahrzehnte. Mehr als die Hälfte aller nationalen Regierungen weltweit haben inzwischen ihre Haushaltsführung umgestellt. Anders als die „klassische“ kamerale Buchführung legen doppisch gebuchte Haushalte den Wertverzehr der öffentlichen Infrastruktur in Form von Abschreibungen offen und zeigen bereits absehbare zukünftige finanzielle Verpflichtungen auf. Dies soll für mehr Transparenz, intergenerative Gerechtigkeit und eine Fokussierung auf öffentliche Leistungen und Ressourcen sorgen.

Grundvoraussetzung für die Nutzung der Doppik ist die vollständige Bewertung des öffentlichen Vermögens. Die erstmalige Erfassung aller Straßen, Gebäude und sonstigen Investitionsgüter ist mit einem enormen personellen, zeitlichen und finanziellen Aufwand verbunden. Für viele Anlagegüter fehlen schlicht Marktpreise und das Vermögen kann nur grob geschätzt werden. So soll die Einführung der Doppik in Frankreich auf Ebene der Zentralregierung insgesamt über zehn Jahre rund 1,5 Mrd. Euro gekostet haben (European Commission, 2013). Internationale Organisationen wie die OECD, der Internationale Währungsfonds oder auch die Europäische Union sehen jedoch langfristige Vorteile und empfehlen trotz der höheren Kosten nachdrücklich die Doppik (Ridder et al., 2005; Flynn et al., 2016; OECD und IFAC, 2017). Seit

(c) Der/die Autor(en) 2020. Open Access: Dieser Artikel wird unter der Creative Commons Namensnennung 4.0 International Lizenz (https:// creativecommons.org/licenses/by/4.0/deed.de) veröffentlicht.

Open Access wird durch die ZBW - Leibniz-Informationszentrum Wirtschaft gefördert. der Staatsschuldenkrise wird auf europäischer Ebene zudem die Einführung einheitlicher doppischer Rechnungslegungsstandards (European Public Sector Accounting Standards, EPSAS) in allen EU-Mitgliedstaaten vorangetrieben (Hilgers et al., 2018). Diese sollen verpflichtend für alle staatlichen Ebenen eingeführt werden. Hierfür rechnet eine Studie von PricewaterhouseCoopers (2014) im Auftrag der Europäischen Kommission für Deutschland mit Reformkosten von bis zu 2,4 Mrd. Euro. Der Bundesrechnungshof (2017) hält diese Schätzungen für nicht belastbar und geht von deutlich höheren Kosten aus.

Prof. Dr. Désirée I. Christofzik ist Professorin für öffentliche Finanzwirtschaft an der Hochschule des Bundes für öffentliche Verwaltung in Brühl.

Florian Dorn ist Vorstandsreferent und Doktorand am ifo Institut in München.

Stefanie Gäbler ist wissenschaftliche Mitarbeiterin und Doktorandin am ifo Institut in München.

Christian Raffer ist wissenschaftlicher Mitarbeiter an der Hertie School Berlin sowie Doktorand an der Freien Universität Berlin.

Dr. Felix Rösel ist wissenschaftlicher Mitarbeiter an der Dresdner Niederlassung des ifo Instituts. 
Erstaunlicherweise stützten sich die Empfehlungen für diese umfassende und aufwendige Umstellung der Haushaltsführung allein auf theoretische Überlegungen, nicht jedoch auf gesicherte empirische Evidenz. Drei unabhängig voneinander entstandene kausal-analytische Studien haben nun die fiskalischen Auswirkungen der DoppikEinführung im öffentlichen Sektor am Beispiel deutscher Kommunen evaluiert. Diese sind bereits vor einigen Jahren zur doppelten Buchführung übergegangen. Die Autoren der drei Studien vergleichen hier ihre Ergebnisse und kommen trotz unterschiedlicher Ansätze, Zeiträume und Untersuchungseinheiten zu sehr ähnlichen und teils überraschenden Schlussfolgerungen.

\section{Veräußerung des „Tafelsilbers“ lohnt sich in der Doppik weniger}

Mit der New-Public-Management-Bewegung kam in den 1980er Jahren der Vorschlag auf, die aus dem Privatsektor bekannte doppelte Buchführung auf den öffentlichen Sektor zu übertragen. Befürworter dieser in Deutschland als "Doppik" bekannt gewordenen Idee versprechen sich transparentere, effizientere und generationengerechtere finanzpolitische Entscheidungen (Budäus, 2009). Dieselbe Politikmaßnahme kann sich dabei in den beiden Systemen sehr unterschiedlich auswirken. Während in der Kameralistik der Fokus auf den jährlichen Zahlungsströmen liegt, zielt die Doppik auf das Reinvermögen ab. Sie stellt also wie in Unternehmensbilanzen den Vermögenswerten die Schulden gegenüber. Damit berücksichtigt sie neben Abschreibungen auch die implizite Verschuldung, die etwa durch Pensionsverpflichtungen entsteht. Sowohl der durch Abschreibungen abgebildete Wertverlust als auch Rückstellungen für absehbare finanzielle Verpflichtungen bleiben in der Kameralistik hingegen unberücksichtigt, da sie während des Betrachtungszeitraums nicht zu Auszahlungen führen.

Ein deutlicher Unterschied tritt auch bei der Veräußerung von Vermögen zu Tage. Eine durch den Verkauf eines Vermögensgegenstandes verursachte Einzahlung kann in der Kameralistik prinzipiell zum Ausgleich des Haushalts genutzt werden - unabhängig vom erzielten Preis. In der Doppik wird das höhere Finanzvermögen vollständig durch ein geringeres Vermögen an Sachanlagen ausgeglichen, sofern der erzielte Preis nicht vom Buchwert abweicht. VeräuBerungen des „Tafelsilbers“ zugunsten eines kurzfristigen Haushaltsausgleichs lohnen sich also nicht in jedem Fall.

Durch die Abbildung des Ressourcenverbrauchs äußern Befürworter der Doppik die Hoffnung auf nachhaltigere Haushaltsentscheidungen und eine bessere Steuerung des öffentlichen Haushalts. Schließlich ergibt sich ein weiterer Vorteil der Doppik im Zusammenspiel mit Ausgliederungen. Nutzen Kernhaushalt und öffentliche Un- ternehmen vergleichbare Systeme, sind konsolidierte Darstellungen einfacher.

\section{Rund drei Viertel aller deutschen Kommunen buchen} doppisch

In Deutschland werden aktuell der Bundeshaushalt und die Mehrheit der Länderhaushalte nach kameralen Standards aufgestellt und ausgeführt. ${ }^{1}$ Auf kommunaler Ebene wird hingegen bereits seit fast zwei Jahrzehnten mit der Doppik experimentiert. Die Innenminister der Länder verständigten sich im Jahr 2003 grundsätzlich auf die Einführung eines doppischen Haushalts- und Rechnungssystems auf kommunaler Ebene (IMK, 2003). Die Bundesländer setzten diese Vereinbarung jedoch in höchst unterschiedlicher Weise um. Während z.B. alle Gemeinden in Nordrhein-Westfalen und Rheinland-Pfalz die Doppik bis zum Jahr 2009 einführen mussten, lief die Frist zur Umstellung für die Kommunen in Baden-Württemberg erst am 1. Januar 2020 aus. Lediglich in Bayern, Thüringen und Schleswig-Holstein können die Gemeinden bis heute ihr Buchungsmodell frei wählen. Insgesamt bucht mit rund $75 \%$ die Mehrzahl der etwa 12.000 Gemeinden und Gemeindeverbände in Deutschland inzwischen doppisch (vgl. Abbildung 1). Zwei Ausnahmen sind die Freistaaten Bayern und Thüringen - in diesen Ländern nutzt nur ein Bruchteil der Kommunen die Doppik.

Ein wesentlicher Grund hierfür könnte der hohe Umstellungsaufwand sein, der sich beispielsweise bei der Aufstellung der Eröffnungsbilanz ergibt. Für diese muss im Rahmen einer Inventur erstmalig das gesamte Vermögen erfasst und bewertet werden. Gleichzeitig dürfte das komplexere Rechnungswesen einen dauerhaften Mehraufwand begründen. Zudem hat das vergleichsweise einfache kamerale System durchaus auch weitere Vorteile. So können die Ansätze aus dem Haushaltsplan laufend mit den tatsächlichen Zahlungen verglichen werden. Dies erleichtert die Kontrolle. Letztlich scheinen bislang auch bei politischen Entscheidungsträgern noch kamerale Denkweisen vorzuherrschen - selbst wenn bereits doppisch gebucht wird (Bundesrechnungshof, 2017).

\section{Aktuelle Evaluationsstudien zu den Auswirkungen der Doppik auf die Finanzpolitik}

Es stellt sich also die Frage, ob sich die Doppik tatsächlich positiv auf finanzpolitische Entscheidungen auswirkt und ob dies den Umstellungsaufwand oder gar einen dauerhaften Mehraufwand rechtfertigt. Die Auswirkungen

1 Aktuell werden die Länderhaushalte in Bremen, Hamburg und Hessen nach doppischem System erstellt. Nordrhein-Westfalen befindet sich in der Umsetzung. 
der Doppik sind empirisch jedoch nicht ohne weiteres zu identifizieren. Es existierten daher bislang kaum empirische Analysen, die über die deskriptive Perspektive von Befragungen von Kämmerern und Kommunalpolitikern hinausgehen. ${ }^{2}$ Vereinzelte Studien beschäftigen sich mit der Wirkung der Doppik-Einführung auf die Effizienz der kommunalen Dienstleistungserbringung oder auf die Zinshöhe für Kommunalkredite (Gärtner, 2013; Bastida et al., 2014; Lampe et al., 2015). Budgetvariablen, die auch für eine Kosten-Nutzen-Rechnung genutzt werden könnten, wurden bislang aber noch nicht in den Blick genommen.

Von dieser Forschungslücke ausgehend wurden in den vergangenen Monaten unabhängig voneinander drei empirische Studien erstellt, die mithilfe quasi-experimenteller Methoden die Wirkung der Doppik auf die kommunalen Haushalte in Deutschland untersuchen (Christofzik, 2019; Dorn et al., 2020; Raffer, 2020). Alle drei Studien analysieren, wie sich verschiedene Haushaltskennziffern nach der Doppik-Einführung in deutschen Kommunen verändert haben. Als Kontrollgruppe dienen jeweils Kommunen, die (noch) kameral buchen. Auf diese Weise können die drei Studien den kausalen Effekt der doppischen Buchführung herausarbeiten. ${ }^{3}$ Raffer (2020) verwendet in seiner Studie Haushaltsdaten von baden-württembergischen Gemeinden für die Jahre von 2005 bis 2016. Dorn et al. (2020) nutzen in ihrer Analyse Daten von Landkreisen und kreisfreien Städten in Bayern für den Zeitraum von 1995 bis 2016. Sowohl in Bayern als auch in Baden-Württemberg konnten die Kommunen selbst den Zeitpunkt der Umstellung wählen - in Bayern bleibt es den Kommunen sogar vorbehalten, gar nicht auf die Doppik zu wechseln. Raffer (2020) und Dorn et al. (2020) vergleichen doppisch buchende Kommunen mit Kommunen, die (noch) nicht auf die Doppik umgestellt haben. Einen etwas anderen Ansatz wählt Christofzik (2019), die aggregierte Gemeindedaten für alle deutschen Flächenländer für den Zeitraum von 1991 bis 2016 betrachtet. Da die Länder weitgehende Freiheiten in der Umsetzung der Doppik haben, unterscheidet sich der Anteil der doppisch buchenden Gemeinden je

2 Für Deutschland siehe etwa Burth und Hilgers (2014) und Kuhlmann et al. (2008), zudem unter anderem Kobayashi et al. (2016) für Japan, Paulsson (2006) für Schweden und Carlin (2005) für Australien.

3 Die drei Studien stützen ihre Analysen dabei insbesondere auf Difference-in-differences-Analysen, erweitert um die Matching-Methode (Raffer, 2020) oder Event-Study-Analysen (Dorn et al., 2020). Da die Gemeinden - überwiegend innerhalb festgelegter Grenzen - ihren Umstellungszeitpunkt frei wählen konnten, besteht grundsätzlich ein Endogenitätsproblem. Die drei Studien adressieren das Problem der Selbstselektion unterschiedlich. Dorn et al. (2020) vergleichen, ob die Entwicklungen zwischen den Doppik-Umstellern und ihrer Kontrollgruppe bereits zuvor voneinander abwichen und finden parallele Trends im Investitionsverhalten. Raffer (2020) wählt mithilfe eines Matching-Ansatzes vergleichbare Kommunen aus und Christofzik (2019) nutzt aus, dass den meisten Gemeinden von ihren Ländern ein festgelegtes Zeitfenster zur Umstellung vorgegeben wurde, was das Problem der Selbstselektion abmildert.

\section{Abbildung 1 \\ Doppisch buchende Gemeinden und Gemeindeverbände in Deutschland \\ Anteil an allen Gemeinden in \%}

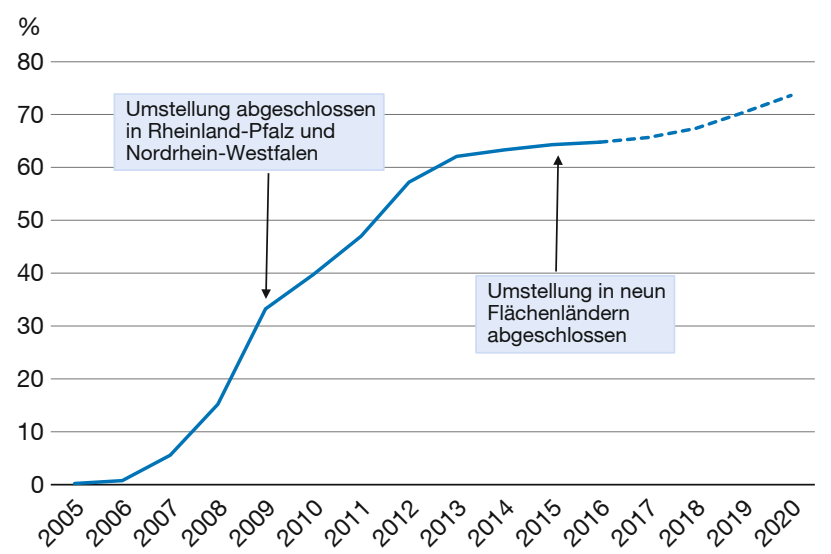

Datenquellen: Christofzik (2019) für die Jahre 2005 bis 2016; eigene Recherchen und Schätzungen für die Folgejahre (gestrichelte Linie); eigene Darstellung.

Bundesland. Diese Variation über die Zeit und zwischen den Ländern wird zur Identifikation genutzt.

\section{Die Doppik wirkt - aber nicht immer wie erwartet}

Trotz unterschiedlicher Datengrundlage und Untersuchungsdesigns gelangen die Studien zu erstaunlich ähnlichen Ergebnissen (vgl. Tabelle 1 für einen detaillierten Überblick). Alle drei Analysen liefern Indizien dafür, dass doppisch buchende Kommunen weniger öffentliches Sachvermögen veräußern als die jeweilige kameral buchende Kontrollgruppe. Überraschenderweise sinken jedoch gleichzeitig tendenziell die Investitionsausgaben wie etwa für Baumaßnahmen oder den Erwerb von Grundstücken und Gebäuden. Wenngleich diese Effekte nicht in allen Studien oder Spezifikationen statistisch signifikant sind, so ist die übereinstimmende Beobachtung eines negativen Effekts auf die öffentlichen Investitionen doch bemerkenswert - lag doch eine Hoffnung auf einer Stärkung der Vermögenssubstanz. Mit der Umstellung auf eine doppische kommunale Haushaltsführung sind in der Tendenz zudem höhere laufende Verwaltungsausgaben verbunden, z. B. für Personal oder Sachaufwand. ${ }^{4}$ Für andere Einnahme- und Ausgabekategorien, den Schuldenstand oder Kassenkredite sowie Variablen, die

4 Den einzigen widersprüchlichen Effekt stellen die Autoren bei der Veräußerung von Finanzvermögen fest: Dorn et al. (2020) finden in Bayern einen Anstieg, Raffer (2020) in Baden-Württemberg einen Rückgang bei der Veräußerung von Finanzvermögen, der jedoch nicht in allen Spezifikationen robust ist. 
Tabelle 1

Kausal-analytische Studien zur Wirkung der kommunalen Doppik: Ausgewählte Ergebnisse

\begin{tabular}{|c|c|c|c|}
\hline Studie & $\begin{array}{l}\text { Christofzik } \\
\text { (2019) }\end{array}$ & $\begin{array}{l}\text { Dorn, Gäbler } \\
\text { und Rösel } \\
\text { (2020) }\end{array}$ & $\begin{array}{l}\text { Raffer } \\
(2020)\end{array}$ \\
\hline Untersuchungseinheit & $\begin{array}{l}\text { Aggregierte } \\
\text { Gemeindeda- } \\
\text { ten auf Ebene } \\
\text { der Bundes- } \\
\text { länder }\end{array}$ & $\begin{array}{l}\text { Landkreise/ } \\
\text { Kreisfreie } \\
\text { Städte in } \\
\text { Bayern }\end{array}$ & $\begin{array}{l}\text { Gemeinden } \\
\text { in Baden- } \\
\text { Württem- } \\
\text { berg }\end{array}$ \\
\hline Beobachtungszeitraum & $1991-2016$ & $1995-2016$ & $2005-2016$ \\
\hline Methodik & $\begin{array}{l}\text { Paneldaten- } \\
\text { modelle, } \\
\text { Difference-in- } \\
\text { differences }\end{array}$ & $\begin{array}{l}\text { Difference- } \\
\text { in-diffe- } \\
\text { rences, } \\
\text { Event-Study }\end{array}$ & $\begin{array}{l}\text { Difference- } \\
\text { in-diffe- } \\
\text { rences mit } \\
\text { Matching }\end{array}$ \\
\hline \multicolumn{4}{|l|}{ Ausgaben } \\
\hline Laufender Sachaufwand & $\Delta$ & $\Delta$ & \\
\hline Personalausgaben & $\Delta$ & O & \\
\hline Investitionsausgaben & $\nabla$ & $\nabla$ & $\boldsymbol{\nabla}$ \\
\hline \multicolumn{2}{|c|}{$\begin{array}{l}\text { Bauausgaben/Investitionen in } \\
\text { unbewegliches Vermögen }\end{array}$} & $\nabla$ & $\boldsymbol{\nabla}$ \\
\hline \multicolumn{4}{|l|}{ Einnahmen } \\
\hline $\begin{array}{l}\text { Veräußerung von Vermö- } \\
\text { gensgegenständen }\end{array}$ & $\nabla$ & $\nabla$ & $\nabla$ \\
\hline $\begin{array}{l}\text { Veräußerung von Finanz- } \\
\text { vermögen }\end{array}$ & & $\Delta$ & $\nabla$ \\
\hline \multicolumn{4}{|l|}{ Schulden } \\
\hline Finanzierungssaldo & O & & \\
\hline Schuldenstand & O & O & \\
\hline \multicolumn{4}{|l|}{ Weitere Auswirkungen } \\
\hline Ausgliederungen & O & & \\
\hline Technische Effizienz & & O & \\
\hline Wahlbeteiligung & & O & \\
\hline
\end{tabular}

Erläuterung: $\Delta / \nabla$ : Signifikanter Effekt. $\Delta / \nabla$ : Teilweise signifikanter Effekt. O: Kein signifikanter Effekt oder unklare Effektrichtung.

Quellen: Christofzik (2019); Dorn, Gäbler und Rösel (2020); und Raffer (2020); eigene Darstellung.

Auswirkungen auf Ausgliederungen oder die Effizienz der kommunalen Leistungen auffangen, lässt sich hingegen in diesen Studien kein systematischer Effekt beobachten. Auch die Wahlbeteiligung bei Kommunalwahlen scheint nicht durch die Umstellung beeinflusst zu werden.

\section{Hintergründe der Investitionszurückhaltung unklar}

Der beobachtbare Rückgang bei der Veräußerung von öffentlichen Vermögensgegenständen entspricht den theoretischen Vorüberlegungen. Bei einem Verkauf entsteht nur dann ein Ertrag, wenn der Gegenstand zu einem Preis veräußert wird, der den Buchwert übersteigt. Werden in der Kameralistik jedoch lediglich die Einzahlungen betrachtet, kann sich die Veräußerung des kommunalen „Tafelsilbers“ zugunsten eines kurzfristigen Haushaltsausgleichs auch zu einem geringeren Preis lohnen. Die Hintergründe höherer Verwaltungsausgaben in doppisch buchenden Kommunen sind ebenfalls naheliegend. Auch von Befürwortern der Doppik wird anerkannt, dass diese Form der Buchführung mit Implementierungskosten sowie einem dauerhaften Mehraufwand einhergeht. Dazu können der Aufwand zur Erstellung der Bilanzen, umfangreichere Schulungsmaßnahmen für das Personal, kostspieligere IT-Lösungen oder externe Beratungskosten gehören.

Etwas überraschend ist indes der konsistent über alle drei Studien hinweg beobachtete negative Effekt auf die öffentlichen Investitionen. Da Investitionen in der Doppik nicht nur zu Auszahlungen führen, sondern zugleich ein Vermögenswert in die Bilanz aufgenommen wird, war eine Hoffnung, dass zusätzliche Investitionen angeregt würden. Die Studienergebnisse zeigen jedoch genau das Gegenteil: die Investitionen gehen in doppisch buchenden Kommunen im Vergleich zur Kontrollgruppe eher zurück. Eine Ursache könnten die höheren Verwaltungsausgaben und die geringeren Einnahmen aus der Veräußerung von öffentlichem Vermögen sein, die den finanziellen Spielraum für neue Investitionen reduzieren. Kommunale Beschäftigte und politische Entscheidungsträger könnten außerdem vor Investitionen zurückschrecken, da sie um den Zusatzaufwand bei der Vermögensbewertung und die Abschreibungen im doppischen System wissen, die sich negativ auf folgende Haushalte auswirken. Auch die fehlende Erfahrung mit dem neuen Buchungssystem könnte eine Zurückhaltung bei der Investitionsneigung erklären. Schließlich könnte die erhoffte höhere Transparenz des Haushalts jedoch auch einfach dazu geführt haben, dass ein Bedarf eben nicht bei neuen Investitionen, sondern der Unterhaltung des bestehenden Vermögens aufgedeckt wurde. Diese Ausgaben würden dem Sachaufwand zugerechnet (Christofzik et al., 2019). Die Bewertung der Nachhaltigkeit von Haushaltsentscheidungen sollte daher nicht allein an der Entwicklung der Investitionen festgemacht werden. Weitere qualitative und quantitative Untersuchungen könnten die Hintergründe des Investitionsrückgangs unter der Doppik jedoch noch näher beleuchten.

\section{Schlussfolgerungen}

Befürworter der Doppik betonen, dass die Einführung des doppelten Haushalts- und Rechnungssystems zu einem effizienteren Ressourceneinsatz führt und nachhaltigere Haushaltsentscheidungen fördert. Damit wird sie teils als Mittel gegen die häufig beklagte Investitionszurückhaltung im öffentlichen Sektor gesehen. Drei kausal-analytische Evaluationsstudien wecken hieran Zweifel. Empirische Analysen von Finanzdaten deutscher Kommunen 
zeigen, dass nach der Umstellung von kameraler auf doppische Buchführung zwar weniger öffentliches Vermögen veräußert, aber auch weniger Geld in neues Vermögen investiert wird - immer im Vergleich zu Kommunen, welche die kamerale Buchführung beibehalten haben. Doppisch buchende Kommunen unterlassen möglicherweise deshalb Investitionen, weil sie den administrativen Aufwand durch die aufwendige Bewertung des neuen öffentlichen Vermögens sowie den jährlichen Aufwand in Form von Abschreibungen scheuen. Es ist daher unklar, ob eine Umstellung von Länder- und Bundeshaushalten auf doppische Standards die öffentlichen Investitionen in Deutschland nicht sogar zusätzlich ausbremsen könnte. Inwiefern es sich bei den beobachteten Effekten um ein dauerhaftes Phänomen handelt, kann anhand der bislang vorliegenden Daten kaum beantwortet werden.

Besonders relevant ist die Aufdeckung der langfristigen Effekte, wenn es darum geht, die potenziellen Folgen der geplanten Einführung europaweit einheitlicher doppischer Buchführungsstandards (EPSAS) zu bewerten. Dabei ist zusätzlich zu beachten, dass Doppik nicht gleich Doppik ist. Die Standards staatlicher Doppik orientieren sich in Deutschland an den Vorschriften des Handelsgesetzbuches, unterscheiden sich aber in einzelnen Punkten zwischen den Ländern. EPSAS lehnt sich hingegen an internationalen Standards an. Auch für die bereits doppisch buchenden Kommunen und Länder wäre ein Umstieg auf harmonisierte europäische Rechnungslegungsstandards daher mit einem nochmaligen Umstellungsaufwand verbunden. Die empirischen Ergebnisse aus den drei Studien verdeutlichen, dass gerade angesichts einer solch umfassenden Reform auch fiskalische Reaktionen bei einer Abwägung von Kosten und Nutzen beachtet werden sollten.

\section{Literatur}

Bastida, F., M. D. Guillamon und B. Benito (2014), Explaining interest rates in local government borrowing, International Public Management Journal, 17(1), 45-73.

Budäus, D. (2009), Manifest zum öffentlichen Haushalts- und Rechnungswesen in Deutschland - Mehr Transparenz, Effektivität und Effizienz in der Politik und Verwaltungen durch ein einheitliches doppisches Haushalts- und Rechnungswesen, ZögU/Journal for Public and Nonprofit Services, 282-318.
Bundesrechnungshof (2017), Bericht nach § 99 BHO über die angestrebte Einführung harmonisierter Rechnungsführungsgrundsätze für den öffentlichen Sektor (EPSAS) in den Mitgliedstaaten der Europäischen Union, Sonderbericht, Bonn.

Burth, A. und D. Hilgers (2014), Cui bono? Depicting the benefits of the new municipal budgeting and accounting regime in Germany, Journal of Business Economics, 84(4), 531-570.

Carlin, T. M. (2005), Debating the impact of accrual accounting and reporting in the public sector, Financial Accountability \& Management, 21(3), 309-336.

Christofzik, D. I. (2019), Does accrual accounting alter fiscal policy decisions? - Evidence from Germany, European Journal of Political Economy, 60, 101805.

Christofzik, D. I., L. P. Feld und M. Yeter (2019), Öffentliche Investitionen: Wie viel ist zu wenig?, Schweizer Monat - Die Autorenzeitschrift für Politik, Wirtschaft und Kultur, 1064, 60-64.

Dorn, F., S. Gaebler und F. Roesel (2020), Ineffective fiscal rules? The effect of public sector accounting standards on budgets, efficiency, and accountability, Public Choice, im Erscheinen.

Ernst \& Young (2012), Overview and comparison of public accounting and auditing practices in the 27 EU Member States, Report prepared for Eurostat.

European Commission (2013), Towards implementing harmonised public sector accounting standards in Member States. The suitability of IPSAS for the Member States, Commission Staff Working Paper SWD, 57.

Flynn, S., D. Moretti und J. Cavanagh (2016), Implementing accrual accounting in the public sector, 2016/06, International Monetary Fund.

Gärtner, J. (2013), Mehr Wirtschaftlichkeit durch Systemwechsel?, LIT Verlag Münster.

Hilgers, D., M. Frintrup und C. Raffer (2018), Sind die EU-Staaten bereit für die EPSAS?, Institut für den öffentlichen Sektor, Berlin.

IMK - Ständige Konferenz der Innenminister und -senatoren der Länder (2003), Sammlung der zur Veröffentlichung freigegebenen Beschlüsse der 173. Sitzung der Ständigen Konferenz der Innenminister und -senatoren der Länder am 21. November 2003 in Jena, Beschluss 18, Anlagen.

Kobayashi, M., K. Yamamoto und K. Ishikawa (2016), The Usefulness of Accrual Information in Non-mandatory Environments: The Case of Japanese Local Government, Australian Accounting Review, 26(2), 153-161.

Kuhlmann, S., J. Bogumil und S. Grohs (2008), Evaluating administrative modernization in German local governments: success or failure of the „new steering model“?, Public Administration Review, 68(5), 851-863.

Lampe, H. W., D. Hilgers und C. Ihl (2015), Does accrual accounting improve municipalities' efficiency? Evidence from Germany, Applied Economics, 47(41), 4349-4363.

OECD und IFAC (2017), Accrual Practices and Reform Experiences in OECD Countries, OECD Publishing.

Paulsson, G. (2006), Accrual accounting in the public sector: experiences from the central government in Sweden, Financial Accountability \& Management, 22(1), 47-62.

PricewaterhouseCoopers (2014), Collection of information related to the potential impact, including costs, of implementing accrual accounting in the public sector and technical analysis of the suitability of individual IPSAS standards. PwC study to inform the impact assessment of EPSAS implementation, Brüssel.

Raffer C. (2020), Accrual Accounting and the Local Government Budget A Matching Evaluation, Arbeitspapier, verfügbar unter SSRN 3547302

Ridder, H. G., H. J. Bruns und F. Spier (2005), Analysis of public management change processes: the case of local government accounting reforms in Germany, Public Administration, 83(2), 443-471.

Title: Does Accrual Accounting Reduce Public Investment? Evidence from Three Recent Evaluation Studies

Abstract: International organisations push for accrual accounting in the public sector. Accrual budgets should reveal the depreciation of public capital stocks and uncover implicit debt liabilities. Three recent studies evaluated the effect of accrual accounting in German local governments - with surprising key findings. Local governments using accrual accounting reduce sales of public assets but also reduce capital spending. Those new findings cast doubt about whether accrual accounting really fosters more sustainable budgeting. JEL Classification: $H 83, H 71, H 72$ 